\title{
A Systematic Approach for Building Change Detection using Multi-Source Data
}

\author{
${ }^{1}$ Abdullah H. Özcan, ${ }^{2}$ Cem Ünsalan, ${ }^{3}$ Peter Reinartz \\ ${ }^{1}$ Tubitak BILLGEM, Turkey \\ ${ }^{2}$ Yeditepe University, Turkey \\ ${ }^{3}$ DLR, Germany \\ (e-mail: abdullah.ozcan@tubitak.gov.tr)
}

\begin{abstract}
Recent sensors give valuable data for remote sensing applications. Among these, building and change detection are important problems. Therefore, researchers worked on these problems using both $2 \mathrm{D}$ and $3 \mathrm{D}$ data. Some previous studies used only 2D data due to their availability. Yet others used either 3D data alone or 2D and 3D data in a joint manner. Besides, some studies only focused on building detection. Yet others used detected building information in change detection. In this study, we focus on 3D change detection based on building information. Therefore, we first detect buildings. At this step, we benefit from both $2 \mathrm{D}$ and 3D data. Then, we locate changes based on these detected buildings. We detect building pixels using panchromatic, multispectral, and Digital Surface Model (DSM) data using a decision tree classifier. Then, we refine the detected building pixels using morphological and shape based operations. Finally, we apply an object based hierarchical change detection method on the refined pixels. We tested our method on 780 buildings and obtained promising results.
\end{abstract}

\section{INTRODUCTION}

Automatic building and change detection are two important problems in remote sensing. As the frequency and strength of recent natural disasters increase, the need for automated systems for building and change detection also increase. Therefore, there are many studies on these either in a separate or joint manner.

The work on building detection can be divided into two different groups. In the first category, researchers used only 2D data (panchromatic, multispectral, or both). The rationale here is that, obtaining the $3 \mathrm{D}$ data is hard and expensive. Therefore, the maximum information from $2 \mathrm{D}$ should be extracted in a cheap and fast manner. In our previous study, we reviewed the literature on 2D building detection [8]. In the second category, researchers used either $3 \mathrm{D}$ data alone or $2 \mathrm{D}$ and $3 \mathrm{D}$ data together [5], [9], [10]. Although 3D data is hard to obtain, it provides valuable information for both building and change detection problems. In this study, we benefit from both $2 \mathrm{D}$ and $3 \mathrm{D}$ data in the building detection part of our method. The 3D data is obtained from the stereo $2 \mathrm{D}$ data pair obtained by the semiglobal matching algorithm [1].

Change detection methods can be divided into two main groups as in building detection. In the first category, there are methods using only 2D data. In these, panchromatic and multispectral data can be used separately or in a joint manner [2]. In the second category, 3D information is taken into account. Adding 3D information to change detection also allows detecting depth changes. In this study, we benefit from both $2 \mathrm{D}$ and $3 \mathrm{D}$ information in the change detection step of our method.

Our systematic approach is composed of three steps. The first one is building detection using classification. Here, we benefit from panchormatic, multispectral, and DSM data from the region to be analyzed. As we obtain the building pixels, we apply a shape based refinement method to extract buildings. Here, we benefit from the box fitting method introduced by Sirmacek and Ünsalan [7]. Finally, we apply object based change detection on the extracted building shapes on bitemporal images. In the following sections, we provide each step in detail.

\section{Classification based Building Detection}

In our system, the first step is building detection. We approach this problem in a standard feature extraction and classification framework. We extract both novel and known eight features first. Then, we feed them to a decision tree classifier. Next, we explain these steps in detail.

\section{A. Features Extracted from Panchromatic Images}

The first extracted feature is the gray value that is obtained directly from the panchromatic image. This feature may seem simple. However, its usage with other features improves the classification performance. The second extracted feature is the variance of the gray values. The variance value for a pixel is calculated by taking a window around it. Our third extracted feature is based on the highest change in a given window. To calculate this feature, we take a window centered around the pixel. Then, we search for the maximum disparity between this pixel and the others in the window. Again, this feature is valuable for detecting building and ground passings.

\section{B. Features Extracted from Multispectral Data}

We used the multispectral data to extract two features. The first one is based on the shadow information. We extract the shadow information using

$$
\text { shadow }=\frac{4}{\pi} \arctan \left(\frac{b-g}{b+g}\right)
$$

where $b$ and $r$ represent the blue and red bands respectively. Shadow information may be used to locate buildings [7]. 
It also gives information for detecting building and ground passings. Therefore, it provides a valuable information.

The second extracted feature using the multispectral data is the angular vegetation index. This index was introduced by us in an earlier work to linearize the NDVI [11]. It can be formulated as

$$
\theta=\arctan \left(\frac{n i r-r}{n i r+r}\right)
$$

where nir and $r$ are the near infrared and red bands respectively. By definition, this index gives information on the vegetation density in a given pixel. Therefore, it can be used to discriminate background and building pixels.

\section{Features Extracted from DSM Data}

We extract three features using DSM data. The first feature is the local height of the pixel. For a selected pixel, $\left(x_{m}, y_{m}\right)$, we define its local height as

$$
I_{h}=D\left(x_{m}, y_{m}\right)-\min (W(x, y))
$$

where, $W(x, y)$ is the subwindow centered around $\left(x_{m}, y_{m}\right)$. This feature may be used to locate buildings based on their height information. Unfortunately, it has a tendency to connect closely located buildings.

The second feature extracted from DSM data is the variance of the height information. Calculating this feature is similar to the variance feature in panchromatic images. The only difference is that, here we benefit from the height information instead of the grayscale values.

The third feature extracted from DSM data is similar to local thresholding. However, in this feature we calculate the local height based on the perpendicular two lines crossing the pixel. Besides, we take the line length as twice the window size in the first feature. This feature is expected to be helpful in locating large buildings on a nearly flat surface.

\section{Classification with a Decision Tree}

The extracted features using panchromatic, multispectral, and DSM data provide different information. Although, one may think that the height information is sufficient for building detection, this is not the case. Tree clusters or natural hillocks may also be taken as building based on their height information. Also closely located buildings is a problem. Here, multispectral data may be of use. However, this may not be sufficient on bare soil. Therefore, using all extracted features is a good strategy for building detection.

In this study, we pick the decision tree structure as the classifier. Decision tree is a supervised learning algorithm [3]. Among other classifiers, it is the simplest and easiest one to interpret. This algorithm is robust to errors and can be used even if the training data contains errors. Decision tree algorithm is in fact a set of rules. From root to leaves, it asks simple comparison questions. At each node, the algorithm builds the tree in a greedy manner and chooses the most informative feature. In this study, we used the ID3 algorithm to select which feature to test at each node. At each step, the entropy of the whole training set is measured if a particular feature for the next step is chosen. In this study, we picked 4300 pixels to train the decision tree. After training, there remains 40 nodes in our tree. In these, every feature is used as a rule to classify pixels as either building or non-building.

\section{Shape BASED Refinement}

Pixel based classification does not take the shape information into account. Since we focus on building change detection in this study, we should embed the shape information to our method. In this section, we provide a way for this purpose. First, we benefit from morphological operations. Then, we use a box fitting algorithm to detect buildings in a more refined manner.

\section{A. Morphological Refinement}

The first step in shape based refinement is using morphological operations. Here, the aim is removing noisy terms before feeding them to box fitting. To do so, we first cluster connected building pixels into five groups based on their pixelwise area. Then, we apply morphological erosion operation via different structuring elements for each group. In other saying, we apply a larger structuring element to larger building pixel blocks. Similarly, we apply different levels of sized structuring elements to each building block. Every step eliminates non building shaped pixel blocks. The remaining pixel blocks are kept as building centers. They will be used in the box fitting algorithm next. As we localize center pixels, then we apply morphological dilation operation to obtain the original sized building pixel blocks.

\section{B. Box Fitting based Refinement}

The second step in shape based refinement is the box fitting method. In the initial version of this method, the box was fit to the target object in one orientation [7]. In the final version of the method, this shortcoming is overcome. A small rectangle is gradually grown starting from the center of the building (seed point). When the rectangles hits the edges of the building the growing stops. This operation is done at different angles. For each angle an energy is calculated which is the sum of minimum distance between virtual building edge pixels and real building edge pixels in perpendicular direction. Then the box with the smallest energy is picked. There are some complex buildings that can not be represented with a single rectangle. Sirmacek et al. also improved the method for complex building shapes [6]. They extracted the skeleton of the building, found junction points and divided building into elongated segments. therefore, they can fit rectangles on each segment of the building.

We also propose similar method for complex building shapes. We represent the building with more than one rectangle. However, we don't find junctions of the building for this purpose. First, we fit a rectangle for the complex shape building. If the ratio of the rectangle area to the building area is less than $90 \%$, then we subtract the rectangle pixels 
from detected building pixels and morphologically clean the remaining small parts. Then, we select the biggest remaining area and find another seed point for fitting another rectangle. We do this iteration until the total area of the rectangles exceeds at least $90 \%$ of the building area. Using this method, complex building shapes can be represented with more than one rectangle.

\section{Change Detection}

There are many studies in the literature using the error-prone bitemporal DSMs in a subtraction process for detecting change [4], [9], [10]. Unfortunately there are many unwanted results of DSM subtraction. Viewing angle of the sensor, illumination, outliers, tree(s) etc. cause unwanted results on the generated DSM. There are also some cases that DSM subtraction would give misleading results. Assume that there are two buildings. Then they are demolished and one new building (different sized in vertical and horizontal) is constructed at the same area. Subtracting DSM will not give the correct result in this case. Therefore, we consider object based change detection in this study.

For object based change detection, we detected building boundaries and represented them with rectangles in the previous section. Using these and related DSM information, we detect changed buildings from bitemporal images. Our systematic approach is as follows. First, we analyse bitemporal images for finding missed buildings in the detection step.

If one building is detected in the first (or second) bitemporal image and not detected in the second (or first) bitemporal image, then we compare the height information for the same area. If the height of the area is similar in both images, then we accept it as a building and correct the building detection step. If a building is not detected in both images, then it will also be not used in change detection. After this correction method, we proceed to building change detection.

We apply our building change detection method on separate buildings. Here, there can be four different change detection category. These are:

- Category 1: No building to building or building to no building change.

- Category 2: Same building with height change.

- Category 3: Two buildings to one building or one building to two buildings change.

- Category 4: Different buildings with height and size change.

Following are the examples for the above categories. If a building is collapsed or a new building is constructed, then it will be in the first category. If the size of a building is not changed, but its height changes then it will be in the second category. If there were two buildings (or one building) in the first time and one building (or two buildings) in the second time at the same area, then it would be in the third category. If a building is collapsed and a new building is constructed with different size, then it will be in the fourth category. Therefore, for each category we use the detected building area and DSM information to find building change category.
Our change detection approach is a hierarchical structure where we detect every change category in an order. First we detect the fourth category changes. If there are buildings in this category, then we discard them in further processing. Then we find the changed buildings in the first category. We discard these also in further processing. Next, we check changed buildings in the second category. Finally, we detect changed buildings in the third category.

\section{EXPERIMENTS}

In this section, we test the strengths and weaknesses of our building and change detection methods. First, we evaluate the building detection performance of our method. Then, we test the change detection performance. In both tests, we benefit from WorldView-2 satellite images and the DSM data extracted from them.

\section{A. Building Detection Performance}

Our bitemporal test images contain 381 buildings in time1 and 399 buildings in time-2 with different size and shape properties. We provide the building detection performance on these images (in terms of True Detection 'TD' anf False Alarm 'FA') in Tables I and II. As can be seen in these tables, the overall true detection performance of our method is $91.3 \%$ and $90.4 \%$ for both times respectively.

TABLE I

BUILDING DETECTION PERFORMANCE ON BITEMPORAL IMAGES IN TIME-1.

\begin{tabular}{lrrr}
\hline Image Name & \# Buildings & TD & FA \\
\hline Istanbul $_{1}\left(t_{1}\right)$ & 141 & 130 & 2 \\
Istanbul $_{2}\left(t_{1}\right)$ & 75 & 68 & 1 \\
Istanbul $_{3}\left(t_{1}\right)$ & 81 & 72 & 2 \\
Istanbul $_{4}\left(t_{1}\right)$ & 84 & 78 & 1 \\
\hline Total & 381 & 348 & 6 \\
\hline
\end{tabular}

TABLE II

BUILDING DETECTION PERFORMANCE ON BITEMPORAL IMAGES IN TIME-2.

\begin{tabular}{lrrr}
\hline Image Name & \# Buildings & TD & FA \\
\hline Istanbul $_{1}\left(t_{2}\right)$ & 146 & 135 & 3 \\
Istanbul $_{2}\left(t_{2}\right)$ & 79 & 72 & 2 \\
Istanbul $_{3}\left(t_{2}\right)$ & 85 & 73 & 2 \\
Istanbul $_{4}\left(t_{2}\right)$ & 89 & 81 & 0 \\
\hline Total & 399 & 361 & 7 \\
\hline
\end{tabular}

We provide the detected buildings as well as the ground truth for four Istanbul images in Fig. 1. We can analyze the performance of our building detection method on these images in detail. Unfortunately, some closely located buildings are missed by our method. In some cases, DSM of the building is not generated. This might be caused by matching errors, interpolation technique or viewing angle of the sensor in the DSM generation step. Therefore, our decision tree algorithm can not detect the building. Some false alarms are mainly because of closely located buildings such that the space between them is detected as a building. 


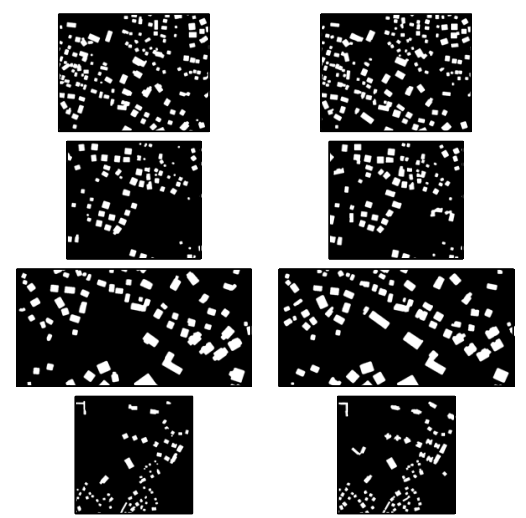

Fig. 1. Detected buildings in time-1 and time-2. Each row corresponds to one bitemporal Istanbul image set (from the first to the fourth). The first column corresponds to time- 1 and the second column to time- 2 .

\section{B. Change Detection Performance}

We analyze the change detection performance of our method based on four categories given above. Based on this definition, we provide the results for each bitemporal image set and change category separately in Table III. In this table, we provide the detected vs the actual number of changes in fractional form. In all four categories, there are total of 31 changes and our system was able to detect 29 of them correctly. There are two missed detections and one false alarm. Missed change detections are because of missed building in the bitemporal image set. The false alarm is caused by very different DSM information of the same building in two images. This may be because of the viewing angle of the sensor.

TABLE III

BUILDING CHANGE DETECTION PERFORMANCE OF OUR METHOD.

\begin{tabular}{lcccc}
\hline Image Set & C1 & C2 & C3 & C4 \\
\hline Istanbul $_{1}$ & $6 / 6$ & $-/-$ & $1 / 1$ & $-/-$ \\
Istanbul $_{2}$ & $9 / 10$ & $-/-$ & $-/-$ & $-/-$ \\
Istanbul $_{3}$ & $5 / 6$ & $1 / 1$ & $-/-$ & $-/-$ \\
Istanbul $_{4}$ & $5 / 5$ & $-/-$ & $-/-$ & $2 / 2$ \\
\hline Total & $25 / 27$ & $1 / 1$ & $1 / 1$ & $2 / 2$ \\
\hline
\end{tabular}

In Fig. 2, we provide the detected changes for all our bitemporal image set. In this figure, we apply following the color code to discriminate change categories: Category one is represented by yellow; category two is represented by magenta; category three is represented by green; category four is represented by red. The reader may check the change detection performance of our method visually by looking at this figure.

\section{Final COMments}

In this study, we proposed a method for building and change detection using multi-source data and shape information. Therefore, we benefit from panchromatic, multispectral, and DSM data. First, we extract novel and known features using these data. Then, we used them in a decision-tree to eliminate non-building areas in pixel wise manner. We then
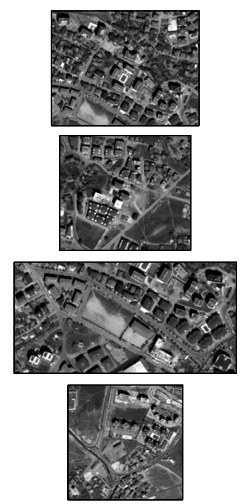
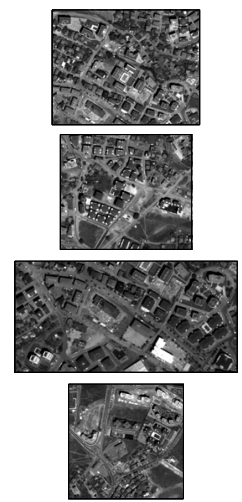

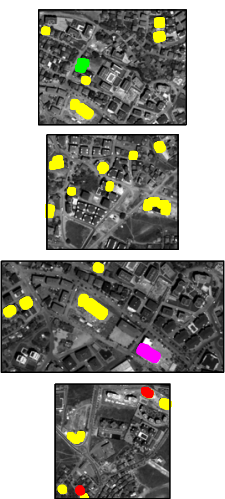

Fig. 2. Change detection results on four bitemporal Istanbul images. First column corresponds to time- 1 , the second column corresponds to time-2, the third column corresponds to the categorized changes.

refined building pixels by shape information. This was for building detection. We tested this step on a large and diverse data set and obtained promising results. Then, we used the detected buildings on change detection. Our change detection method is based on objects. It also has a hierarchical structure. Test results on four bitemporal images show the effectiveness of our method.

\section{REFERENCES}

[1] P. dAngelo and P. Reinartz, "Semi-global matching results on the isprs stereo matching benchmark," in Int. Archives Photogrammetry, Remote Sensing and Spatial Information Sciences, vol. XXXVIII-4/W19, 2011.

[2] M. İlsever and C. Ünsalan, Two Dimensional Change Detection Methods: Remote Sensing Applications. Springer, 2012.

[3] T. M. Mitchell, Machine Learning. McGraw-Hill, 1997.

[4] H. Murakami, K. Nakagawa, H. Hasegawa, T. Shibata, and E. Iwanami, "Change detection of buildings using an airborne laser scanner," ISPRS Journal of Photogrammetry and Remote Sensing, vol. 54, pp. 148-152, 1999.

[5] A. H. Özcan, C. Ünsalan, and P. Reinartz, "Building detection using local features and DSM data," in Proceedings of RAST'13, 2013, pp. 139-143.

[6] B. Sirmacek, P. dAngelo, and P. Reinartz, "Detecting complex building shapes in panchromatic satellite images for digital elevation model enhancement," in ISPRS Workshop on Modeling of Optical Airborne and Space Borne Sensors, 2010.

[7] B. Sirmacek and C. Ünsalan, "Building detection from aerial images using invariant color features and shadow information," in Proceedings of ISCIS'08, 2008.

[8] —, "A probabilistic framework to detect buildings in aerial and satellite images," IEEE Transactions on Geoscience and Remote Sensing, vol. 49, no. 1, pp. 211-221, 2011.

[9] J. Tian, S. Cui, and P. Reinartz, "Building change detection based on satellite stereo imagery and digital surface models," IEEE Transactions on Geoscience and Remote Sensing, vol. 52, no. 1, pp. 406-417, 2014.

[10] J. Tian and P. Reinartz and P. d'Angelo and M. Ehlers "Region-based automatic building and forest change detection on Cartosat-1 stereo imagery," in ISPRS Journal of Photogrammetry and Remote Sensing, 2013.

[11] C. Ünsalan and K. L. Boyer, "Linearized vegetation indices based on a formal statistical framework," IEEE Transactions on Geoscience and Remote Sensing, vol. 42, pp. 1575-1585, 2004.

\section{ACKNOWLEDGMENT}

This work is supported by TUBITAK under project no $110 \mathrm{E} 302$. 